\title{
El giro kantiano de Rawls hacia el hegelianismo
}

\section{( William Farfán Moreno \\ Universidad Santo Tomás, Bogotá, Colombia}

\begin{abstract}
Resumen
El presente artículo discute el giro que hace Rawls desde la interpretación kantiana de la justicia como imparcialidad hacia el hegelianismo. El primer Rawls considera la interpretación kantiana de la justicia como imparcialidad como el argumento más fuerte que sustenta la legitimidad en su idea del contrato social. Respondiendo a las críticas de los comunitaristas (especialmente las de Sandel) Rawls da un giro del kantismo al hegelianismo incorporando dos constructos relevantes que no aparecen en $A$ Theory of Justice: por una parte, el consenso traslapado conectado con el equilibrio reflexivo y, por otra, la razón pública. Así, el segundo Rawls (el del liberalismo político) es más hegeliano que kantiano.
\end{abstract}

\begin{abstract}
This article discusses the Rawlsian turn from the Kantian interpretation of justice as fairness to Hegelianism. The first Rawls considers the Kantian interpretation of justice as fairness as the strongest argument that sustains the legitimacy of his idea of social contract. In responding to the criticisms of communitarians (especially those of Sandel) Rawls make a turn from Kantianism to Hegelianism, introducing two important constructs that don't appear in A Theory of Justice: first, the overlapping consensus connected to reflective equilibrium, and second, public reason. In this way the second Rawls (political liberalism) is more Hegelian than Kantian.
\end{abstract}

\section{Introducción}

Rawls, como respuesta a sus críticas por parte de los multiculturalistas y, esencialmente, de los comunitaristas, ha tenido un punto de inflexión de su teoría de la justicia de inspiración kantiana (primer Rawls) hacia una posición hegeliana que se evidencia en su obra Political Liberalism (segundo Rawls). Allí se pone de manifiesto la influencia hegeliana cuando propone los constructos como mecanismos para responder a las críticas comunitaristas aceptadas por Rawls. A partir de este contexto, el siguiente planteo lo abordaremos como sigue: en primera instancia, se planteará muy brevemente la inspiración kantiana de Rawls en $A$ Theory of Justice y las críticas que surgieron
Palabras clave

rawlsianismo kantianismo comunitarismo hegelianismo

Keywords

Rawlsianism Kantianism Communitarianism Hegelianism 
1. Escribe Kant en la Fundamentación de la metafisica de las costumbres. "Por reino entiendo el enlace sistemático de distintos seres racionales por leyes comunes. Mas como las leyes determinan los fines, según su validez universal, resultará que, si prescindimos de las diferencias personales de los seres racionales y asimismo de todo contenido de sus fines privados, podrá pensarse un todo de todos los fines (tanto de los seres racionales como fines en sí, como también de los propios fines que cada cual puede proponerse) en enlace sistemático; es decir, un reino de los fines, que es posible según los ya citados principios" (Kant, 1995: 47). a partir de allí por parte de los comunitaristas (I); luego se abordarán las respuestas a tales críticas en Political Liberalism (II), en el que se definirá la influencia hegeliana que, indefectiblemente, es en el momento de la eticidad del sistema hegeliano.

\section{El kantismo en Rawls}

La influencia kantiana se puede analizar desde el estudio que hace el filósofo norteamericano John Rawls de La fundamentación de la metafísica de las costumbres de Kant en sus clases sobre la historia de la filosofía moral impartidas en Harvard y que luego fueron compiladas en el texto llamado Lectures on the history of moral philosophy por Barbara Herman. En Lectures... se evidencia la influencia que tuvo el kantismo en las nociones más relevantes de la justicia como imparcialidad de Rawls.

Inicia con la noción kantiana de reino de los fines entendida como el todo sistemático de los fines del conjunto de seres racionales capaces de autogobernarse. Lo esencial en Kant para fundamentar el reino de los fines es alcanzar la buena voluntad para actuar por respeto a la ley moral mediante el imperativo categórico. Ahora bien, Rawls comprende que el imperativo categórico debe construirse a través de un procedimiento en cuatro pasos al que le llama "procedimiento IC": desde la formulación de un imperativo hipotético, pasando por la generalización de la máxima como precepto universal que a su vez deberá transformarse en ley natural humana -análogamente como una ley se convierte en ley universal de la naturaleza - hasta que ésta última constituya un nuevo orden social que Rawls llamará un mundo social ajustado.

Así, la buena voluntad tiene el papel de darle primacía a la justicia, en cuanto que los seres racionales y razonables le encontrarán sentido a la vida en el mundo al respetar la ley moral, asegurando así la moralidad y legitimidad de todo acto frente a otros seres racionales y razonables considerados como fines y no como medios. De esta noción procede la idea análoga de "sociedad bien ordenada" en Rawls entendida como la sociedad efectivamente regulada por una concepción pública de la justicia. No obstante, Rawls al considerar la fundamentación monológica de la base consensual del contrato social en Kant, además fundamenta la sociedad bien ordenada - en donde todos los agentes morales aceptan y son capaces de actuar bajo unos principios de justicia como lo harán los seres racionales y razonables en un posible reino de los fines bajo el imperativo categórico-como un sistema justo de cooperación. De aquí que la justicia como imparcialidad sea considerada una teoría deontológica y no teleológica.

Si en Kant es necesario prescindir de las diferencias personales de los seres racionales y de todo contenido de sus fines privados para pensar en un conjunto de todos los fines (lo que Rawls llama los dos límites de la información), ${ }^{1}$ de la misma manera Rawls habrá de pensar en tales límites de la información para elegir los principios reguladores de la justicia distributiva y por ende de la cooperación de los ciudadanos en la sociedad. Rawls le da el nombre de "posición original con velo de ignorancia"; es tal noción la que va a asegurar que los principios de la justicia no sean elegidos heterónomamente y a posteriori, contaminados por factores empíricos. Lo único que han de tener claro los agentes morales en el momento de decidir los principios son los bienes sociales primarios que le van a imprimir un carácter real y no utópico a la realización de los principios de la justicia, lo que en Kant sería una "máxima del interés común" que son las necesidades humanas verdaderas. En suma, el imperativo categórico kantiano con sus características que pueden derivarse de su carácter sintético a priori es análogo a los principios de la justicia, que en su momento de elegirse, deben tener en cuenta las nociones de la razón práctica (lo racional y lo razonable) junto con las concepciones de ciudadano y sociedad. 
No obstante lo anterior, Rawls ${ }^{2}$ introduce también dos sub-constructos referidos a los principios de la justicia: ${ }^{3}$ el primero es lo que él llama "orden lexicográfico consecutivo" (a serial or lexical order, aunque el término correcto para Rawls es lexicographical), cuyo orden serial significaría que ningún principio ha de intervenir en una situación particular mientras no hayan sido satisfechos los primeros. El primer principio definirá el ordenamiento constitucional de la sociedad y el segundo la distribución de la riqueza; en este caso el primer principio, el de igual libertad, será situado jerárquicamente en un orden anterior quedando como regulador de las desigualdades económicas y sociales sujetas a él. El segundo sub-constructo es el "equilibrio reflexivo", cuyo fin es el de darle validez a los principios en la medida que paulatinamente se vayan comprobando al contraponerlos con las convicciones propias y proporcionar orientaciones concretas en situaciones particulares. ${ }^{4}$

De aquí que la concepción de la "justicia como imparcialidad" es la de la justicia procedimental pura, esto es, la elección argumentada de los principios en condiciones de igualdad y libertad para las partes, y no la de justicia procedimental perfecta que supone un principio de justicia anterior a la argumentación de la misma. Es aquí donde debe tenerse en cuenta los conceptos de autonomía racional y autonomía plena. El primero viene dado por la equidad que el velo de ignorancia garantiza a las partes. Con él, las partes no poseen información específica sobre su situación, tan sólo el conocimiento de los bienes primarios sociales. El segundo, la autonomía plena, se define como racional en cuanto el provecho personal de cada participante, sin embargo, aunque esté bajo la estructura de la posición original, el criterio de orientación será lo razonable, de tal manera que éste subordina lo racional. Como lo racional pertenece a la razón práctica empírica y lo razonable a la razón práctica pura, entonces se colige que la razón práctica empírica estará subordinada por la razón práctica pura, dando así razón a la característica del constructivismo kantiano: la prioridad de lo justo (lo razonable) sobre lo bueno (lo racional).

Por último, en el parágrafo 40 de $A$ Theory of Justice (Rawls, 1971: 221- 227), Rawls es claro con su teoría de la justicia como imparcialidad afirmando que ella es una interpretación kantiana. En él se destacan varios puntos relacionados con los principios de la justicia en concordancia con el imperativo categórico, a saber: primero, cuando se actúa autónomamente es porque los principios de su acción son elegidos por la persona como la expresión más adecuada de su naturaleza como ser libre y racional; segundo, los principios de las acciones no dependen de contingencias naturales, sociales o particulares, por tal motivo son sintéticos a priori; por lo tanto (tercera idea), los principios de justicia son también imperativos categóricos; y cuarto, la posición original puede ser considerada como una interpretación procesal de la concepción kantiana de autonomía y del imperativo categórico dentro del sistema de una teoría empírica. Estas ideas, que son claves para entender la influencia kantiana, logran dar un alcance y una visión filosófica más amplia sobre la teoría de la justicia de Rawls. Es así como se logra comprender el giro pragmático a la teoría kantiana haciendo de la autonomía y la libertad los elementos constitutivos de la sociedad civil.

No obstante, la inspiración kantiana en la obra de Rawls fue motivo de fuerte críticas por la postura ahistórica, metafísica y universalista de su teoría de la justicia. Tales críticas lo motivaron a avanzar en su propuesta y trabajar en su liberalismo político. A continuación se presentarán las críticas y luego, a raíz de éstas, el giro que da el filósofo hacia el hegelianismo.

\section{El giro de Rawls hacia el hegelianismo}

En la década de los ochenta, filósofos tales como Charles Taylor, Michael Walzer, Alasdair McIntyre y Michael Sandel realizaron unas fuertes críticas al liberalismo
2. Esencialmente desarrollados en Rawls (1971: 36-40).

3. Los dos principios rezan así en Justicia como Equidad: "a) cada persona tiene el mismo derecho irrevocable a un esquema plenamente adecuado de libertades básicas iguales que sea compatible con un esquema similar de libertades para todos; $y$ b) las desigualdades sociales $y$ económicas tienen que satisfacer dos condiciones: en primer lugar, tienen que estar vinculadas a cargos y posiciones abiertos a todos en condiciones de igualdad equitativa de oportunidades y, en segundo lugar, las desigualdades deben redundar en un mayor beneficio de los miembros menos aventajados de la sociedad (el principio de diferencia)" (Rawls 2002: 73). 4. "It is an equilibrium because at last our principles and judgments coincide; and it is reflective since we know to what principles our judgments conform and the premises of their derivation. At the moment everything is in order. But this equilibrium is not necessarily stable. It is liable to be upset by further examination of the conditions which should be imposed on the contractual situation and by particular cases which may lead us to revise our judgments" (Rawls, 1971: 18). 
individualista. Concretamente rechazaron la concepción ahistórica de un individuo cargado de derechos con anterioridad a la de su marco social, político y cultural. Un liberalismo que propende a este individualismo liquida los valores culturales o comunitarios. Precisamente, según los comunitaristas, en este error cayó el liberalismo de Rawls en su teoría de la justicia. Otra fuerte crítica era que su teoría de la justicia tenía un carácter tan abstracto que no lograba percibir la existencia de otras doctrinas razonables o comprensivas, e incluso, plantearon que su interpretación kantiana de la justicia como equidad era otra doctrina comprensiva entre muchas más.

Por ello, Rawls dio un giro significativo a su teoría de la justicia introduciendo dos conceptos desarrollados en Political Liberalism cuyo objetivo, además de responder a sus críticos, fue tomar distancia frente al kantismo - considerado como una doctrina comprehensiva entre otras varias-y considerar un constructivismo político y no moral, que le permitiera no considerarlo como otra doctrina razonable o comprensiva. De aquí que complemente la "justicia como imparcialidad" con dos constructos adicionales: "el consenso traslapado" y "la razón pública".

Como la idea es atender el giro hacia el hegelianismo de Rawls que se dio en el contexto de las críticas comunitaristas, entonces analizaremos este giro a partir de este punto basándonos en la postura que toma Rorty. Este autor es quien realiza una defensa al liberalismo rawlsiano de las críticas comunitaristas a partir del sub-constructo del "consenso traslapado"/"equilibrio reflexivo". Es a partir de éste como se comprenderá aquella prioridad de la justicia sobre las concepciones del bien, o como lo titula Rorty en uno de sus ensayos, la "prioridad de la democracia sobre la filosofía".

Rorty inicia la defensa a Rawls contra las críticas de los comunitaristas, en especial las críticas de Sandel, haciendo remembranza de la política liberal de Thomas Jefferson: "Los ciudadanos de una democracia jeffersoniana pueden ser tan religiosos o irreligiosos como plazcan siempre que no sean fanáticos" (Rorty, 1996: 239). Rorty, a propósito de este comentario, emplea tres conceptos fundamentales que abren paso a una discusión en una forma de gobierno de corte liberal: "religioso", "irreligioso" y "fanáticos". Los dos primeros formarían parte de un sistema democrático liberal, ya que el hecho de considerarse religioso o no a un ciudadano en un sistema liberal no afectaría en gran medida una democracia constitucional debido al pluralismo y a la tolerancia. Por otra parte, sería diferente el discurso si entre esos grupos religiosos o irreligiosos el fanatismo fuera el foco de la sociedad en las decisiones políticas. En el primer caso, Rorty identificará a los pragmatistas, cuyos representantes son John Dewey y John Rawls; y en el segundo caso (los fanáticos), se encontrarán los "absolutistas". Respecto de éstos, afirman que todo ser humano tiene todas las creencias necesarias para la virtud cívica y que se derivan de una facultad humana universal, es decir, de una conciencia "cuya posesión constituye específicamente la esencia de cada ser humano" (Rorty, 1996: 240). Por otro lado, los pragmáticos afirman que los individuos encuentran en sus conciencias creencias relevantes, pero en aras de la convivencia general deben ser sacrificadas, esto es: que lo político debe prevalecer sobre las convicciones religiosas y morales.

De estas nociones se desprenden diferentes concepciones de los "derechos humanos". Por una parte, el absolutista hablará de derechos humanos inalienables, argumentando que en todo lugar, época y cultura, los miembros de nuestra especie han tenido los mismos derechos. Por otra parte, si el pragmático considera la referencia a los "derechos" un intento de disfrutar de los beneficios de la metafísica sin asumir las responsabilidades correspondientes, necesitará de un criterio que logre distinguir entre la conciencia individual que se respeta en un estado liberal posmoderno y el grado de conciencia que termine condenada por el mismo liberalismo como "fanática". Según Rorty, este criterio será algo relativamente local, es decir, que se define 
conforme a la tradición de una comunidad particular o al consenso de una cultura específica: "De acuerdo con esta concepción, lo que pasa por racional o por fanático es relativo al grupo ante el que creemos justificarnos - al cuerpo de creencias comunes que determina la referencia al término nosotros" (Rorty, 1996: 241). En esta última concepción - la pragmática - se encuentra, según Rorty, John Rawls, para quien es irrelevante la formulación de una idea de la verdad que sirva como base para la estructura política. Sin embargo, Rorty además de identificar estas dos vertientes del liberalismo, la absolutista y la pragmática, identifica una tercera teoría social: el denominado "comunitarismo" cuyos representantes son Alasdair McIntyre, Charles Taylor, Robert Bellah y Michael Sandel. Éste último es el crítico comunitarista más relevante de la teoría de la justicia de Rawls. Rorty mostrará discrepancias con las críticas de Sandel y verá en la teoría de Rawls más un liberalismo pragmatista que un liberalismo universalista abstracto.

El comunitarismo rechaza el individualismo racional de la Ilustración y la idea de "derechos" al igual como lo hace el pragmatismo, pero discrepa con éste en que el comunitarismo además pone en juicio "las instituciones y la cultura de los Estados democráticos supervivientes" (Rorty, 1996: 242). Rorty resume en tres ideas esta tesis o discrepancia con los comunitaristas: a) “...la predicción empírica de que ninguna sociedad que deja de lado la idea de verdad moral ahistórica de la manera despreocupada en que Dewey recomendó puede sobrevivir". Es decir, que la sociedad que planteaba la Ilustración puede sobrevivir sin los argumentos universalistas de la filosofía moral liberal. Luego, b) “(...) el tipo de ser humano creado por las instituciones y la cultura liberal es indeseable", y c) “(...) las instituciones políticas presuponen una doctrina sobre la naturaleza del ser humano y que - al contrario del racionalismo de la Ilustración - esta doctrina debe poner en evidencia la índole esencialmente histórica del yo" (Ibídem). Tales posturas de los comunitaristas son críticas directas que hacen a los liberales absolutistas y que llevaron a Sandel a realizar su rechazo al supuesto universalismo rawlsiano. De esta manera escribe Sandel:

[E]n la teoría de la justicia de Rawls se encuentra implícita una concepción del sujeto moral que tanto modela los principios de la justicia como es modelado a su imagen a través del medio de la posición original. Es esta concepción la que me propongo iluminar y explorar. Si puede hacerse explícita de alguna manera, ayudaría no solamente a resolver las perplejidades relativas al rango de la posición original, sino también a evaluar los presupuestos centrales de la concepción como un todo. Mientras que el argumento principal de Rawls tiende a asumir la naturaleza del sujeto moral como dada, y a llegar a los principios de la justicia a través de la posición original, me propongo a trabajar en el sentido opuesto: tomar los principios de la justicia como provisionalmente dados y volver sobre nuestros pasos hacia la naturaleza del sujeto moral. Al hacerlo, asumo que estoy volviendo sobre los lineamientos de un argumento del siguiente tipo: asumiendo que somos seres capaces de justicia, y más precisamente, seres para quienes la justicia es primaria, debemos ser criaturas de un cierto tipo, relacionados con las circunstancias humanas en cierta forma. ¿Qué debe ser verdad acerca de un sujeto para quien la justicia es la primera virtud? ¿Y cómo se incorpora la concepción de este tipo de sujeto en la posición original? (Sandel, 2000: 71)

Es claro entonces que para Sandel la posición original de Rawls es un mecanismo que al asegurar autonomía y libertad a los ciudadanos racionales y razonables, le imprime un carácter universalista relegando toda circunstancia contextual y presuponiendo una doctrina sobre la naturaleza humana. Sandel plantearía un proceso diferente al de Rawls: partiría no de un sujeto moral dado que sea capaz de deliberar los principios de justicia a través de la posición original, sino que partiría de los principios de justicia provisionalmente como dados para volver al sujeto moral con el fin de 
acomodarlos a las circunstancias humanas de las "criaturas de un cierto tipo". No obstante, en Rorty ronda una pregunta sobre esta interpelación de Sandel y concretamente sobre la tercera afirmación de los comunitaristas (la c.): ¿la democracia liberal necesita justificación filosófica alguna, como por ejemplo, una justificación sobre la naturaleza humana? En este caso, según la interpretación de Rorty (Rorty, 1996: 243), Sandel cree que Rawls antepone una filosofía universalista en la posición original, y en razón de ella, ubica luego la política o las instituciones. Es decir, Sandel piensa que Rawls justifica filosóficamente las instituciones políticas sin tener en cuenta el yo histórico de las culturas, pero Rorty va a negar rotundamente esta perspectiva de Sandel y va a argumentar que Rawls muestra cómo puede funcionar la democracia liberal sin presupuestos filosóficos universales.

Rawls, según Rorty, soslaya temas como una naturaleza humana ahistórica, la naturaleza del yo, la motivación de la conducta moral y el sentido de la vida humana. Estos temas son irrelevantes para la política, por eso la solución no está en saber cuál es la mejor postura filosófica que pudiera fundamentar la teoría política. De aquí procede la idea de Rorty de porqué la actitud historicista y anti-universalista de Rawls, pues como cita el primero textualmente sobre el segundo: "la filosofía en cuanto búsqueda de la verdad sobre un orden metafísico y moral independiente no puede... proporcionar una base operativa y común a una concepción política de la justicia en una sociedad democrática" (Rawls citado en Rorty, 1996: 246). ${ }^{5}$ Por lo tanto, como la filosofía no puede ser la base de una sociedad democrática, entonces es necesario recoger las convicciones consolidadas de una democracia liberal tales como la tolerancia religiosa y el rechazo de la esclavitud con el fin de organizar las intuiciones implícitas en estas convicciones de una concepción de la justicia. Es esta postura de Rawls la inflexión hacia el hegelianismo y a su vez lo que le da un giro pragmático a la teoría kantiana. Lo que realmente consolidará esta postura hegeliana de Rawls será el "equilibrio reflexivo" del "consenso traslapado".

En el equilibrio reflexivo del consenso traslapado, Rawls deja en el ámbito privado las convicciones filosóficas y religiosas con el fin de separarlas de la política, pues ellas podrían ser una amenaza para la libertad y la justicia si en algún caso se llegara a imponer una convicción interna o una legislación moral sobre lo político o la legislación jurídica. El recurso del equilibrio reflexivo de Rawls asegurará la tolerancia filosófica y religiosa, y como método para las discusiones de política social, servirá para equilibrar las intuiciones: "Mientras sigamos pensando que las conclusiones políticas requieren fundamentos extra políticos - es decir, mientras pensemos que el método de equilibrio reflexivo de Rawls no es suficientemente bueno-desearemos una explicación de la autoridad de aquellos principios generales" (Rorty, 1996: 250). De esta forma, con el equilibrio reflexivo de Rawls, Rorty plantea un contextualismo en donde la justicia, como primera virtud de una sociedad, se derivará del acuerdo entre la deliberación de individuos que son herederos de las mismas tradiciones históricas y se encuentran enfrentados con los mismos problemas de su propia comunidad.

En contraste, Sandel atribuye a Rawls la construcción de cierta concepción del yo haciendo uso de la metafísica para legitimar la política liberal. Un planteamiento de este tipo tenderá a carecer de todo tipo de pragmatismo y conducirá indefectiblemente a un absolutismo, ya que - siguiendo el análisis de Rorty sobre la crítica de Sandella teoría rawlsiana según la cual "la justicia es la primera virtud de las instituciones sociales", requiere del respaldo de la pretensión metafísica, por la cual "sobre la base de una teología invertida, lo más esencial para nuestra personalidad no son los fines que elegimos, sino nuestra capacidad para elegirlos. Y esa capacidad se halla en un yo que debe ser anterior a los fines que elige" (Sandel citado en Rorty, 1996: 253). De este modo, Sandel en El liberalismo y los Limites de la justicia (Sandel, 2002: 76-83) considerará que los fines son previos a la persona, que ya están fijados cuando 
nace por la cultura a la cual pertenece. Esto será contrario al liberalismo político de Rawls, pues para éste último el "yo" es anterior a los fines que mediante él se afirman. Según Sandel esto es lo característico de la ética deontológica cuya autonomía de la persona será anterior a los fines; pero no es así en Sandel: los fines son anteriores a la autonomía de la persona y sencillamente esta autonomía deberá ser respetuosa con los valores y fines de la comunidad. Por eso concluye Sandel que Rawls mediante la posición original lo que hace es ocuparse de la naturaleza del "yo", de cuáles son las características de quienes elegirán los principios que no entren en conflicto con los fines de la comunidad y de los ciudadanos, más no se ocupa de la naturaleza de los deseos u objetivos del "yo": "Se refiere al sujeto de los intereses y fines, y no al contenido de éstos, cualesquiera que sean" (Sandel, 2000: 77).

Desde luego Rorty va a rechazar este planteamiento de Sandel. Él considera que la lectura que se debe hacer de A Theory ofJustice debe ser desde la perspectiva política y no metafísica. Si se realiza conforme a lo político, se verá que la afirmación de Rawls acerca de que el yo está antes que los fines que se afirman con él, no se debe interpretar como el yo ajeno y diferente al conjunto de creencias y deseos que posee. Cuando Rawls afirma en $A$ Theory of Justice que "no debemos intentar dar forma a nuestra vida, remitiéndonos en primer lugar al bien definido de modo independiente" (citado en Rorty, 1996: 253), Rorty interpreta el "debemos", no en razón de la naturaleza intrínseca de la moralidad ni tampoco porque la capacidad de elección constituye la esencia de la personalidad, sino en razón a que el "nosotros" - quienes practican la tolerancia religiosa y tienen un gobierno constitucional - ponen a la libertad por encima de la perfección. Es esta idea la que va a tener en cuenta Rorty:

Rawls no desea una "visión deontológica completa", que explicase por qué debemos dar a la justicia la prioridad sobre nuestra concepción del bien. Está completando las consecuencias de la afirmación de que es aquélla [la justicia] la que tiene prioridad, no sus presupuestos. Rawls no se interesa por las condiciones de la identidad del yo, sino solamente por las condiciones de la ciudadanía en una sociedad liberal (Rorty, 1996: 258).

Así pues, las condiciones de la ciudadanía en una sociedad liberal son las que van a determinar la estructura básica de la sociedad y no serán las diferentes concepciones del bien que se tengan acerca de la justicia, ni tampoco lo será la misma filosofía. La verdad y la filosofía serán irrelevantes para la democracia política y cuando entren en conflicto, la democracia es la que tendrá prioridad sobre la filosofía.

Ahora bien, ¿de qué manera el equilibrio reflexivo rawlsiano es de carácter hegeliano? Es precisamente en la eticidad de Hegel donde Rawls es hegeliano. Allí Hegel percibe al hombre tal cual en su contexto histórico y con sus instituciones propias. No un hombre moderno producto de una realidad abstracta, sino de su propia historia, cultura, religión y creencias, pero que, producto de la racionalidad moderna, se ha ido concibiendo separado de las instituciones. Un hombre separado de las instituciones jamás podrá ejercer y disfrutar realmente de su libertad. Un hombre que desee ejercer y disfrutar de la libertad separada de las instituciones, es un hombre fragmentado y aislado de su propia comunidad. Por ello, sólo en el momento de la eticidad hegeliana, es donde el hombre es realmente libre: es un hombre concreto que piensa en la libertad concreta.

Ya en las Lecciones sobre la filosofía de la historia universal (Hegel, 2005: 128), el filósofo alemán afirma que la historia universal es la exposición del espíritu, ${ }^{6}$ de cómo el espíritu labora por llegar a saber lo que es en sí: se sabe y se quiere libre. Según Hegel, la razón del espíritu y por tanto el fin último del mundo sería: “...que el Espíritu tenga conciencia de su libertad y que de este modo su libertad se realice” (Hegel, 2005: 129).
6. Walter Jaeschke afirma que si bien antes de Hegel se entendía "espíritu" como el pneuma neotestamentario -es decir: desde la postura teológica一 o bien que en tiempos del mismo filósofo alemán ni siquiera la noción tenía una postura filosófica concisa, Hegel lo convierte en el centro de sus reflexiones filosóficas y lo comprenderá más bien desde su estructura interna, esto es: ser referencia a sí mismo. Para explicar Hegel este continuo movimiento del Espíritu en la realidad, hace uso del método dialéctico, es decir, de un método que comprende el movimiento como evolución interna del Espíritu. De esta forma, como lo explicará Jaeschke, el Espíritu que se referencia a sí mismo desde su estructura interna se comprenderá desde tres momentos: 1 ) como la Psicología rationalis que pretendía probar la simplicidad y la inmortalidad de Ima bajo el nombre de Espíritu subjetivo: 2) pero que forma frente a él una estructura propia que hace necesaria su existencia a través del derecho, la moralidad y la eticidad: el Espiritu objetivo; no obstante 3) el Espíritu se manifiesta más allá de la vida social y se eleva entonces al plano de la vida espiritual a través del arte, la religión y la filosofía. Éste es el Espíritu absoluto. Así pues, según lo anterior, el Estado se encuentra en el momento del Espíritu objetivo, es decir, en el momento en que el Espíritu puede objetivarse, manifestándose en las comunidades humanas. Sólo si se objetiva la libertad en las acciones humanas, la libertad se vuelve concreta y real. Es por ello que cuando el Espíritu se encarna en el Estado se realiza la libertad siendo capaz de conciliar los intereses de todos los ciudadanos y actuar conforme al bien común (Cfr. Jaeschke, 1998). 
7. ["El Estado es la realidad efectiva de la libertad concreta; pero la libertad concreta consiste en que la individualidad personal y sus intereses particulares tienen tanto su perfecto desarrollo y el reconocimiento de su derecho para sí (en el sistema de la familia y de la sociedad civil), cuanto, por una parte, trascienden por sí mismos en el interés universal $y$, por otra parte, lo reconocen con saber y voluntad como su propio espiritu sustancialy actúan para él como su finalidad última, de manera que ni lo universal tiene violencia y se consuma sin el interés particular, el saber y el querer, ni los individuos viven meramente para el interés supremo como personas privadas, sin que a la vez quieran en lo universal y para lo universal y tengan una actividad consciente de esta finalidad. El principio de los Estados modernos tiene ese poder inmenso y la profundidad de dejar perfeccionarse hasta el extremo autónomo de la particularidad personal y a la vez retrotraerlo a la unidad sustancial y así conservar en él mismo a esa unidad."; Hegel (2000: 308-309) (N. del E.)].
El material donde se realiza y verifica la libertad es en el Estado. Es allí donde la libertad se hace objetiva y se realiza positivamente. A propósito, Rawls hace un análisis interesante de esta idea hegeliana a partir del siguiente párrafo de la filosofía del derecho de Hegel (parágrafo 260):

[a] The state is the actuality of concrete freedom. But concrete freedom requires that personal individuality [Einzelheit] and its particular interests should reach their full development and gain recognition of their right for itself (within the family and civil society),

[b] and also that they should, on the one hand, pass over of their own accord into the interest in the universal, and on the other, knowingly and willingly acknowledge this universal interest even as their own substantial spirit and actively pursue it as their ultimate end.

[c] The effect of this is that universal does not attain validity or fulfillment without the interest, the knowledge and the volition of the particular, and that individuals do not live private persons merely for these particular interests without at the same time directing their will to a universal end [in und für das Allgemeine wollen] and acting in conscious awareness of this end.

[d] The principle of modern states has enormous strength and depth because it allows the principle of subjectivity to attain fulfillment in the self-sufficient extreme of personal particularity, while at the same time bringing it back to substantial unity and so preserving this unity in the principle of subjectivity itself (Rawls, 2000: 354). ${ }^{7}$

En el primer párrafo (a) — según Rawls - cuando se indica la "realidad efectiva de la libertad concreta", es en el sentido de que permite a los individuos satisfacer sus intereses particulares que se desarrollan dentro del marco permitido por los derechos y deberes establecidos por la familia y la sociedad civil, además de estar protegidos por el imperio de la ley: "The state as a whole is the framework of basic political and social institutions that, together with the family and civil society, enable citizens, who are members of the state, to attain their freedom" (Rawls, 2000: 355).

Respecto al segundo punto (b), es interesante la forma como Hegel entiende los intereses particulares. Cuando Hegel indica que los intereses se convierten por sí mismos en interés general, es porque entiende que la sociedad se mantiene unida en virtud de un sentido de orden razonable regulado por una concepción de la justicia basada en el bien común y no por la persecución de los fines particulares. Entendemos por ello que Hegel cuando cita en Lecciones sobre la filosofía de la historia universal que en el Estado la libertad se hace objetiva y se realiza positivamente, no significa que la voluntad subjetiva del individuo se realice y se goce mediante la voluntad general, siendo ésta última un medio para la voluntad subjetiva del individuo; ni tampoco significa que el Estado sea una reunión de hombres, en la que la libertad de todos tiene que ser limitada; sino que el Estado es el lugar donde los intereses particulares logran verse e interiorizarse por todos como intereses comunes. Al respecto comenta Rawls:

What raises human life above the workaday bürgerliche world is the recognition of the universal interest of all citizens in participating in and maintaining the whole system of political and social institutions of the modern state that makes their freedom possible. Citizens knowingly and willingly acknowledge this universal (collective) interest as their own, and they give it the highest priority (Rawls, 2000: 354).

Esta "máxima prioridad" se convertirá en el fin último de las acciones. 
En el párrafo tercero (c) Rawls indica que en Hegel, ese interés universal tiene validez sólo por el interés, el saber y la aceptación de los ciudadanos individuales de la sociedad civil. Así como éstos están preocupados por sus intereses particulares, del mismo modo deben estar preocupados por lo universal que está basado en una concepción de la justicia. De esta forma, tanto la voluntad como las acciones conscientes de los ciudadanos están orientadas hacia este fin. Esta última parte es lo que en Rawls sería la razonabilidad, esto es, la capacidad de cooperar con el bien común y los fines de la justicia. El deber para Hegel de estar preocupados por lo universal -en términos del interés común- es el mismo deber rawlsiano de que los ciudadanos sean razonables. Así, las acciones que los ciudadanos realicen en términos políticos (participar en los asuntos públicos), deben tener presente el deber de civilidad, esto es, el deber de ser razonables.

Y en cuanto al último párrafo (d) comenta Rawls:

Hegel creía que el hecho de que la constitución moderna permitiera la plena libertad en la sociedad civil daba una fuerza enorme al Estado, siempre que el interés universal (colectivo) de los ciudadanos fuera reconocido por ellos y ellos mismos le otorgaran prioridad en su vida política. ${ }^{8}$

Aquí el papel de la razón pública propuesta en Political Liberalism es central para interpretar del mismo modo la influencia hegeliana en el segundo Rawls. La razón pública se modelará, según Rawls, con la posición original de tal forma que los principios de justicia elegidos por los representantes de los ciudadanos bajo tal constructo, tendrán como resultado el reconocimiento público y mutuo entre todos los ciudadanos sobre tales principios. En otras palabras, cuando los ciudadanos eligen racional y razonablemente los principios de justicia que regularán su sociedad en la posición original con el uso de la posición original con velo de ignorancia, ellos expresarán su disponibilidad para ofrecer los justos términos de cooperación social entre sí y acatar los principios elegidos. Esto es lo que Rawls denomina el "criterio de reciprocidad". El resultado de este reconocimiento (principio de reciprocidad) es nada menos que la Constitución, con la fuerza que le otorga Hegel en la vida política de los ciudadanos.

Así pues, para concluir, decimos que son tres razones por las cuales podemos considerar un giro del universalismo kantiano al hegelianismo en Rawls. En primer lugar cuando, al responder a las críticas comunitaristas, pone en el constructivismo político los constructos del "consenso traslapado" y el "equilibrio reflexivo". 9 La otra razón por la cual se permite concluir que el segundo Rawls es hegeliano, es el constructo de la "razón pública", que aparece en Political Liberalism. Esto no es más que el interés universal de los ciudadanos individuales de la sociedad civil para validarlo y aceptarlo: como se mencionó anteriormente, así como los ciudadanos están preocupados por sus intereses particulares, del mismo modo deben estar preocupados por lo universal que está basado en una concepción de la justicia. En este sentido, la razón pública adquiere esta connotación hegeliana en tanto que se debe modelar con la posición original en la que los ciudadanos razonablemente elegirán los principios que regularán su sociedad y cuyo resultado concreto, además del reconocimiento público y mutuo entre ciudadanos de estos principios, es la Constitución y la Corte Constitucional como tribunales de la razón pública. Por último, el giro hacia el hegelianismo de Rawls derivada de las dos anteriores, es la de suponer que los ciudadanos son concebidos como seres racionales y razonables. Es la razonabilidad la que permitirá la existencia de una sociedad justa y bien ordenada en términos rawlsianos. Sin esa capacidad moral de concebirnos como ciudadanos capaces de cooperar en el interés universal en términos hegelianos, sería impensable un mundo en el que podamos ser realmente libres.
8. "Hegel believed that the fact that the modern constitution allowed for full freedom in civil society gave enormous strenght to the state, provided that the universal (collective) interest of citizens was recognized and given priority by them in their political life" (Rawls, 2000: 356).

9. Los constructos son trabajados por Rawls en sus dos obras principales: en A Theory of Justice Rawls propone tres constructos, a saber: 1) la "posición original" y un sub-constructo de ésta que es el "velo de ignorancia"; 2) los dos principios básicos de su teoría de la justicia y un sub-constructo de éstos que es el orden lexicogáfico; y 3) el "equilibrio reflexivo". Y en Political Liberalism Rawls agrega otros dos constructos que son el resultado de las críticas que surgieron a raíz de su teoría de la justicia: 1) el "consenso traslapado" (o solapado) en el que retoma el equilibrio reflexivo y que se analizó con Rorty y 2) la idea de la "razón pública". 


\section{Q Bibliografía}

"Hegel, G. W. F. (2000). Rasgos fundamentales de la filosofía del derecho (Trad. Vásquez, E.). Madrid: Biblioteca Nueva.

" Hegel, G. W. F. (2005). Lecciones sobre la Filosofía de la Historia Universal (Trad. Gaos, J.). Madrid: Tecnos.

» Hegel, G. W. F. (2009). Fenomenología del Espíritu. México: Fondo de Cultura Económica.

» Jaeschke, W. (1998). Hegel. La conciencia de la modernidad. Madrid: Akal.

» Kant, I. (1995). Fundamentación de la metafísica de las costumbres. México: Porrúa.

» Rawls, J. (1971). A Theory of Justice. Cambridge: Harvard University Press.

» Rawls, J. (1993). Political Liberalism. New York: Columbia University Press.

" Rawls, J. (2000). Lectures on the history of moral philosophy (Ed. Barbara Herman). Cambridge: Harvard University Press.

" Rawls, J. (2002). Justicia como Equidad. Una reformulación (ed. Erin Kelly). Barcelona: Paidós.

» Rorty, R. (1996). Objetividad, relativismo y verdad. Escritos filosóficos 1. Barcelona: Paidós.

»Sandel, M. (2000). El liberalismo y los límites de la justicia. Barcelona: Gedisa. 